\title{
Aetiology and outcome of acute diarrhoea in children with severe acute malnutrition: a comparative study
}

\author{
Abhishek Jain ${ }^{1}$, Dheeraj Shah ${ }^{1, *}$, Shukla Das ${ }^{2}$, Rumpa Saha ${ }^{2}$ and Piyush Gupta ${ }^{1}$ \\ 'Department of Paediatrics, University College of Medical Sciences and Guru Teg Bahadur Hospital, Delhi 1 10095, \\ India: ${ }^{2}$ Department of Microbiology, University College of Medical Sciences and Guru Teg Bahadur Hospital, Delhi, India
}

Submitted 29 January 2019: Final revision received 16 June 2019: Accepted 8 July 2019; First published online 8 November 2019

\begin{abstract}
Objective: To compare the microbiological profile, clinical course and outcome of acute diarrhoea in children aged $<5$ years having severe acute malnutrition (SAM) with those of children having normal nutritional status.

Design: Cross-sectional comparative study.

Setting: Tertiary-care hospital catering mainly to the urban poor of East Delhi, India.

Participants: Children aged $<5$ years ( $n$ 140; seventy with SAM (cases) and seventy with normal anthropometry (controls)) with acute diarrhoea (duration $<14 \mathrm{~d}$ ). Stool samples were collected for conventional culture, microscopy, acid-fast staining, rotavirus and Cryptosporidium antigen detection, and subtyping of diarrhoeagenic Escherichia coli (DEC). We followed-up these children for persistent diarrhoea and subsequent diarrhoeal episode in the next 3 months.

Results: Rotavirus was detected in six (9\%) cases and in fifteen (21\%) controls $(P=0.03 ;$ OR $=0.34 ; 95 \%$ CI $0 \cdot 12,0.94)$. DEC was isolated significantly more in cases compared with controls (93 v. 64\%; $P<0.001$; OR $=7.25 ; 95 \%$ CI 2.57 , 20.4). Cryptosporidium was detected in seven (10\%) cases and five (7\%) controls. Total duration of diarrhoea and percentage change in weight after resolution of diarrhoea were comparable between cases and controls. At 3-month follow-up, number of subsequent episodes of diarrhoea and persistent diarrhoea were comparable between the two groups.

Conclusions: Rotavirus was found significantly less frequently, whereas DEC was detected more frequently in children with SAM in comparison to non-malnourished children. To further reduce diarrhoea-related mortality, preventive and therapeutic interventions need to be designed against organisms causing diarrhoea in children with SAM.
\end{abstract}

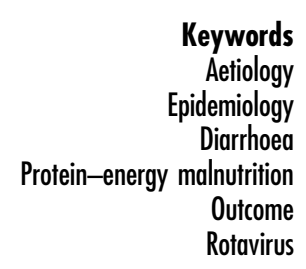

Diarrhoea and severe acute malnutrition (SAM) are the key contributors to mortality among children aged $<5$ years (under-5s), especially in low- and middle-income countries ${ }^{(1-3)}$. Both increase the incidence of one another. Diarrhoea leads to malabsorption, increased catabolism, nutrient sequestration and decreased dietary intake, which results in malnutrition; whereas malnutrition results in impaired immune function and barrier action that further increases the chances of infection ${ }^{(4)}$. Malnourished children are at a greater risk of diarrhoea than well-nourished children because of weak local IgA response ${ }^{(5,6)}$, and also at a much higher risk of diarrhoea-related death and complications such as worsening of malnutrition and delayed recovery $^{(7)}$.
Aetiology, risk factors, outcomes and complications of diarrhoea in children are well known. Rotavirus is implicated as the most important causative agent among under-5 children with diarrhoea ${ }^{(8,9)}$. However, there is a paucity of studies focusing on aetiology and outcomes in children with SAM having acute diarrhoea. India, belonging to the group of low- and middle-income countries, faces sanitation as one of the biggest challenges: about $64 \%$ of the poor rural population lacked improved sanitation facilities and $48 \%$ of the rural population defecated openly in the year $2012^{(10)}$. With the widespread use of oral rehydration salts, zinc and standardized protocols for management of diarrhoea, diarrhoea-related mortality has reduced significantly and most deaths are now occurring in those with 
SAM $^{(11)}$. Hence, efforts must focus on knowing the aetiology and profile of diarrhoea in children with SAM to help in designing and refining the treatment guidelines in these children. We conducted the present study with the objectives of determining the aetiology and outcomes of acute diarrhoea in children with SAM and to compare them with those in well-nourished children.

\section{Methods}

The present cross-sectional analytical study (with enrolment of cases and controls) was conducted in the Departments of Paediatrics and Microbiology of a hospital catering mainly to the urban poor population of East Delhi and affiliated to a medical college in New Delhi, India, between January 2016 and March 2017. An approval from the institutional ethical committee was obtained. Informed consent was obtained from the parent or guardian of every participating child.

\section{Study population}

\section{Inclusion criteria}

We enrolled children aged between 1 and 59 months presenting to the paediatric outpatient department or emergency services with acute watery diarrhoea (duration $<14 \mathrm{~d})^{(12)}$. Children with SAM (weight-for-height/length Z-score (WHZ) <-3 according to WHO reference standards or bipedal oedema or mid-upper arm circumference (MUAC) $<11.5 \mathrm{~cm}$ ) were enrolled as cases ${ }^{(13)}$ and normally nourished children (WHZ $>-2$ according to WHO reference standards and no bipedal oedema and MUAC $>12.5 \mathrm{~cm}$ ) served as controls. Cases and controls were matched for gender and age group ( $<3$ months, 3-6 months, 6-12 months, 1-3 years, 3-5 years).

\section{Exclusion criteria}

Children with coexisting chronic systemic illness (e.g. chronic heart, kidney, haematological, liver or gastrointestinal disease), known cases of HIV or immune suppression, preterm birth ( $<37$ weeks of gestation), and children receiving antibiotics, probiotics or any other indigenous medication within the last 1 week before enrolment were excluded.

\section{Clinical assessment}

A detailed clinical history was obtained, with focus on nature of diarrhoea, vomiting, fever and abdominal pain. Hydration status was assessed at the time of enrolment as per WHO criteria ${ }^{(12)}$. A general physical examination and systemic examination were performed for every participant at the time of enrolment. Every participating child was assessed for weight (digital weighing scale, sensitivity 5 g; SECA, Germany), length/height (infantometer/stadiometer, sensitivity $1 \mathrm{~mm}$; SECA) and MUAC (measuring tape, sensitivity $1 \mathrm{~mm}$; SECA) using standard techniques ${ }^{(14)}$.
These data were interpreted using WHO reference growth charts and $Z$-scores were calculated using WHO Anthro software (version 3.2.2). Anthropometry was repeated and analysed at resolution of diarrhoea.

\section{Microbiological assessment}

Stool samples (freshly passed, 1-2 g) were collected in widemouth containers or by using a sterile applicator to transfer them to the container after collecting on a clean mackintosh. Part of the stool samples was used for rotavirus antigen detection by the EpiTuub ${ }^{\circledR}$ Fecal Rotavirus Antigen Test Kit (Epitope Diagnostic, USA). Another part of the stool samples was concentrated by the formalin-diethyl ether sedimentation method. The resulting samples were examined microscopically by Kinyoun's acid-fast staining for detection of Cryptosporidium oocyts. The samples were also preserved in $10 \%(\mathrm{v} / \mathrm{v})$ formalin (at room temperature) for antigen detection of Cryptosporidium, using the CoproELISA Cryptosporidium Antigen Detection Kit (Savyon Diagnostics, Israel) as per the manufacturer's instruction. Saline-iodine preparations of the stools were examined under a microscope for the presence of ova, cysts, pus cells and erythrocytes. Stool samples were cultured for enteric pathogens on a battery of media and incubated at $37^{\circ} \mathrm{C}$ overnight to be identified by conventional laboratory techniques. Stool cultures positive for enteric pathogenic bacteria and/or Escherichia coli were noted. Lactose-fermenting colonies from MacConkey agar, confirmed biochemically as $E$. coli, were picked up for DNA extraction (HiYield Genomic DNA Kit; Real Biotech Corporation, Taiwan) and multiplex PCR. Specific primers were used to identify diarrhoeagenic E. coli (DEC): enteropathogenic E. coli (EPEC), enterotoxigenic E. coli (ETEC) and enteroaggregative E. coli (EAEC), which were procured from Genei, India. DNA amplification was performed on a thermocycler and analysed by electrophoresis on $1 \%(\mathrm{w} / \mathrm{v})$ agarose gel.

\section{Patient management}

Children were hospitalized depending upon the clinical condition, state of hydration and state of malnutrition. Participating children were treated as per the WHO standard treatment protocols ${ }^{(12,15)}$. Well-nourished children were supplemented with oral zinc. Parents were counselled about exclusive breast-feeding and complementary feeding. Importance of environmental sanitation, personal hygiene, hand washing and family planning was also emphasized. Enrolled children were followed-up every fortnight in the follow-up clinic of the hospital over the next 3 months for recurrence of diarrhoea.

\section{Outcome variables}

The primary objective of our study was to find the frequency of detection of rotavirus, as the causative agent of acute watery diarrhoea, in both study groups. The secondary objectives were to find the proportion of 
children having Cryptosporidium-, Shigella- and E. colirelated diarrhoea in both study groups. We also noted the percentage change in weight after the resolution of diarrhoeal illness, duration of diarrhoeal illness (from the day of onset of loose stools $>3$ times/d to no loose stools in the last $24 \mathrm{~h}$ ), and the percentage of children developing persistent diarrhoea (duration $>14 \mathrm{~d}$ ) and/or recurrence during the 3-month follow-up.

\section{Sample size}

The proportion of well-nourished children (controls) with detection of rotavirus antigen in stools was estimated to be $0 \cdot 3^{(16)}$. Assuming the proportion to be $0 \cdot 1$ among severely malnourished children (cases), we required sixty-two cases and sixty-two controls to reject the null hypothesis of no difference in proportion of rotavirus detection in the two groups with $80 \%$ power and $\alpha$ error of 0.05 . Therefore, we planned to enrol seventy under- 5 children with acute watery diarrhoea in each group allowing for $10 \%$ loss of analysable samples.

\section{Statistical analysis}

Frequency of aetiological agents and the number of children developing persistent diarrhoea in both study groups were compared using the $\chi^{2}$ test or Fisher's exact test. The percentage change in weight, duration of diarrhoeal illness and number of repeat episodes of diarrhoea were compared between cases and controls using Student's $t$ test (unpaired).

\section{Results}

We enrolled 140 under-5 children with acute diarrhoea, including seventy with SAM and seventy who were normally nourished. The median age of participating children was 10 months. Eighty (57.1\%) children were less than 1 year of age. Table 1 compares the clinical profile and anthropometry between the two groups.

Table 2 compares the microbiological agents between cases and controls. We observed rotavirus in twenty-one enrolled children; it was found in a significantly lower proportion of cases than controls. DEC was isolated in 110 enrolled children; the proportion was significantly higher in cases. Out of all the subtypes, EAEC was isolated in a significantly higher number of cases compared with controls. Frequency of mixed infection with other DEC was also high. EAEC was detected in seventy-three children, out of whom twenty-four children had isolated EAEC and rest, forty-nine, had mixed infection. Similarly, EPEC and ETEC were detected in fifty-three and fifty children, out of whom twenty-one and twelve had isolated infection and rest, thirty-two and thirty-eight children, respectively, had mixed infection with other DEC. Twelve children (8.6\%) had stool samples positive for Cryptosporidium
Table 1 Comparison of characteristics between cases and controls at enrolment: children aged $<5$ years, East Delhi, India, January 2016-March 2017

\begin{tabular}{|c|c|c|c|c|c|}
\hline \multirow[b]{2}{*}{ Parameter } & \multicolumn{2}{|c|}{ Cases $(n 70)$} & \multicolumn{2}{|c|}{ Controls $(n 70)$} & \multirow[b]{2}{*}{$\begin{array}{c}P \\
\text { value }\end{array}$} \\
\hline & $\begin{array}{l}\text { Mean } \\
\text { or } n\end{array}$ & $\begin{array}{l}\text { SD } \\
\text { or } \%\end{array}$ & $\begin{array}{l}\text { Mean } \\
\text { or } n\end{array}$ & $\begin{array}{l}\text { SD } \\
\text { or } \%\end{array}$ & \\
\hline $\begin{array}{l}\text { Age (months) } \\
\text { mean and SD }\end{array}$ & $16 \cdot 1$ & 14.65 & $14 \cdot 4$ & $12 \cdot 04$ & 0.474 \\
\hline \multicolumn{6}{|l|}{ Sex, $n$ and $\%$} \\
\hline Males & 29 & 41.4 & 30 & $42 \cdot 9$ & 0.864 \\
\hline $\begin{array}{l}\text { Duration of } \\
\text { diarrhoea at } \\
\text { enrolment (d), } \\
\text { mean and SD }\end{array}$ & $2 \cdot 0$ & $1 \cdot 77$ & 1.9 & 1.53 & 0.759 \\
\hline $\begin{array}{l}\text { No. of stools in } \\
24 \mathrm{~h} \text {, mean and } \\
\text { SD }\end{array}$ & $10 \cdot 4$ & 3.41 & $10 \cdot 3$ & $2 \cdot 80$ & 0.829 \\
\hline \multicolumn{6}{|c|}{ Type of stools, $n$ and \% } \\
\hline Watery stools & 55 & $78 \cdot 6$ & 52 & 74.3 & \\
\hline Mucoid stools & 12 & $17 \cdot 1$ & 13 & $18 \cdot 6$ & 0.826 \\
\hline Blood in stools & 3 & $4 \cdot 3$ & 5 & $7 \cdot 1$ & \\
\hline Vomiting, $n$ and $\%$ & 40 & $57 \cdot 1$ & 39 & 55.7 & 0.865 \\
\hline Fever, $n$ and $\%$ & 32 & $45 \cdot 7$ & 36 & 51.4 & 0.499 \\
\hline $\begin{array}{l}\text { Abdominal pain, } n \\
\text { and } \%\end{array}$ & 17 & $24 \cdot 3$ & 11 & $15 \cdot 7$ & 0.205 \\
\hline \multicolumn{6}{|c|}{ Hydration status, $n$ and $\%$} \\
\hline No dehydration & 6 & $8 \cdot 6$ & 7 & 10 & \\
\hline $\begin{array}{l}\text { Some } \\
\text { dehydration }\end{array}$ & 30 & $42 \cdot 9$ & 28 & 40 & 0.923 \\
\hline $\begin{array}{l}\text { Severe } \\
\text { dehydration }\end{array}$ & 34 & $48 \cdot 6$ & 35 & 50 & \\
\hline \multicolumn{6}{|c|}{ Anthropometry at enrolment, mean and SD } \\
\hline Weight $(\mathrm{g})$ & 5922 & 2169 & 8482 & 2575 & $<0.001$ \\
\hline MUAC (cm) & $10 \cdot 9$ & 1.00 & 13.4 & 0.50 & $<0.001$ \\
\hline WAZ & $-4 \cdot 13$ & 1.00 & -1.00 & 1.06 & $<0.001$ \\
\hline WHZ & -4.05 & 0.90 & -0.70 & 0.80 & $<0.001$ \\
\hline
\end{tabular}

Cases, children having severe acute malnutrition and acute diarrhoea; controls, anthropometrically normal children with acute diarrhoea; MUAC, mid-upper arm circumference; WAZ, weight-for-age Z-score; WHZ, weight-for-length/height $Z$-score.

antigen detection by ELISA. Out of these twelve samples, eight were also positive on acid-fast staining of the concentrated stool.

We found a statistically significant difference in weight at resolution of diarrhoea and WHZ at resolution of diarrhoea between cases and controls (Table 3), similar to the significant difference observed in WHZ at enrolment. We also observed that the percentage change in weight and change in WHZ at resolution were similar and comparable in both study groups. Persistent diarrhoea was observed in six participants, three each in cases and controls. We were able to follow-up 130 enrolled children for recurrence of diarrhoea and its characteristics. The mean (SD) duration of follow-up was $66.5(19.3) \mathrm{d}$ in cases and $66.7(21.5) \mathrm{d}$ in controls and the mean (SD) number of diarrhoeal episodes during follow-up was $1.7(1.6)$ in cases and 1.5 (1.2) in controls.

\section{Discussion}

In the present cross-sectional study, rotavirus was detected significantly less frequently among SAM cases with acute 
Table 2 Microbiological comparison between cases and controls: children aged $<5$ years, East Delhi, India, January 2016-March 2017

\begin{tabular}{|c|c|c|c|c|c|c|c|}
\hline \multirow[b]{2}{*}{ Aetiological agent } & \multicolumn{2}{|c|}{$\begin{array}{l}\text { Cases } \\
(n 70)\end{array}$} & \multicolumn{2}{|c|}{$\begin{array}{l}\text { Controls } \\
(n 70) \%\end{array}$} & \multirow[b]{2}{*}{$P$ value } & \multirow[b]{2}{*}{ OR } & \multirow[b]{2}{*}{$95 \% \mathrm{Cl}$} \\
\hline & $n$ & $\%$ & $n$ & $\%$ & & & \\
\hline Rotavirus & 6 & $8 \cdot 6$ & 15 & 21.4 & 0.033 & 0.34 & $0.12,0.94$ \\
\hline Cryptosporidium & 7 & $10 \cdot 0$ & 5 & $7 \cdot 1$ & 0.55 & 1.45 & $0.44,4.78$ \\
\hline DEC & 65 & $92 \cdot 9$ & 45 & $64 \cdot 3$ & $<0.001$ & $7 \cdot 25$ & $2 \cdot 57,20 \cdot 4$ \\
\hline EPEC & 21 & - & 32 & - & 0.556 & 0.51 & $0.25,1.02$ \\
\hline EAEC & 43 & - & 30 & - & 0.028 & $2 \cdot 12$ & $1.08,4.17$ \\
\hline ETEC & 29 & - & 21 & - & 0.158 & 1.65 & $0.82,3.31$ \\
\hline Vibrio cholera & 5 & - & 9 & - & 0.546 & 0.69 & $0.21,2.29$ \\
\hline Other bacteria (total) & 15 & - & 9 & - & 0.178 & & \\
\hline Shigella spp. & 3 & - & 1 & - & & & \\
\hline Salmonella spp. & 1 & - & 1 & - & & & \\
\hline Klebsiella spp. & 5 & - & 4 & - & & & \\
\hline Pseudomonas spp. & 4 & - & 1 & - & & & \\
\hline Citrobacter spp. & 2 & - & 2 & - & & & \\
\hline Protozoa and helminths (total) & 13 & - & 13 & - & 1.000 & & \\
\hline Giardia & 11 & - & 6 & - & & & \\
\hline Ascaris & 1 & - & 3 & - & & & \\
\hline Hookworm & 1 & - & 1 & - & & & \\
\hline Hymenolepis nana & 1 & - & 1 & - & & & \\
\hline Enterobius vermicularis & 0 & - & 2 & - & & & \\
\hline
\end{tabular}

Cases, children having severe acute malnutrition and acute diarrhoea; controls, anthropometrically normal children with acute diarrhoea; DEC, diarrhoeagenic Escherichia coli; EPEC, enteropathogenic E. coli; EAEC, enteroaggregative E. coli; ETEC, enterotoxigenic E. coli.

Table 3 Comparison of parameters at diarrhoea resolution between cases and controls: children aged $<5$ years, East Delhi, India, January 2016-March 2017

\begin{tabular}{|c|c|c|c|c|c|}
\hline \multirow[b]{2}{*}{ Parameter at resolution } & \multicolumn{2}{|c|}{$\begin{array}{l}\text { Cases } \\
(n 70)\end{array}$} & \multicolumn{2}{|c|}{$\begin{array}{c}\text { Controls } \\
(n 70)\end{array}$} & \multirow[b]{2}{*}{$P$ value } \\
\hline & $\begin{array}{l}\text { Mean } \\
\text { or } n\end{array}$ & $\begin{array}{l}\mathrm{SD} \\
\text { or } \%\end{array}$ & $\begin{array}{l}\text { Mean } \\
\text { or } n\end{array}$ & $\begin{array}{l}\text { SD } \\
\text { or } \%\end{array}$ & \\
\hline Weight $(\mathrm{g})$, mean and SD & 6175 & 2215 & 8875 & 2679 & $<0.001$ \\
\hline WHZ, mean and SD & -3.58 & 0.85 & -0.20 & 0.80 & $<0.001$ \\
\hline $\begin{array}{l}\text { Total duration of } \\
\text { diarrhoea (d), mean } \\
\text { and SD }\end{array}$ & 4.7 & 3.5 & $4 \cdot 1$ & $3 \cdot 4$ & 0.318 \\
\hline $\begin{array}{l}\text { Percentage change in } \\
\text { weight, mean and SD }\end{array}$ & 4.67 & $2 \cdot 55$ & 4.48 & $2 \cdot 01$ & 0.616 \\
\hline $\begin{array}{l}\text { Change in WHZ, mean } \\
\text { and SD }\end{array}$ & 0.47 & 0.24 & 0.50 & 0.23 & 0.520 \\
\hline \multirow{3}{*}{$\begin{array}{l}\text { Persistent diarrhoea, } \\
n \text { and } \%\end{array}$} & 3 & $4 \cdot 3$ & 3 & $4 \cdot 3$ & $1 \cdot 0$ \\
\hline & \multicolumn{2}{|c|}{$\begin{array}{l}\text { Cases } \\
(n 65)\end{array}$} & \multicolumn{2}{|c|}{$\begin{array}{c}\text { Controls } \\
(n 65)\end{array}$} & \\
\hline & Mean & SD & Mean & SD & $P$ value \\
\hline Duration of follow-up (d) & 66.5 & $19 \cdot 3$ & $66 \cdot 7$ & 21.5 & 0.949 \\
\hline $\begin{array}{l}\text { Episodes of acute } \\
\text { diarrhoea in } 3 \text { months* }\end{array}$ & $1 \cdot 7$ & $1 \cdot 6$ & 1.5 & $1 \cdot 2$ & 0.395 \\
\hline
\end{tabular}

Cases, children having severe acute malnutrition and acute diarrhoea; controls, anthropometrically normal children with acute diarrhoea; WHZ, weight-for-length/ height Z-score.

*Many children were not completely followed up for 3 months; episodes of diarrhoea in them were averaged to 3 months.

diarrhoea in comparison to controls (well-nourished children) with acute diarrhoea. We observed high rates of isolation of DEC for both cases and controls. DEC isolation was significantly higher in children with SAM. Out of all three subtypes, EAEC was significantly higher in cases compared with controls. Cryptosporidium and Vibrio cholera detection was comparable between cases and controls. We did not observe any significant difference in the duration of illness, percentage change in weight, percentage change in WHZ and incidence of persistent diarrhoea between the two groups.

In our study, we found rotavirus in $15 \%$ of total children enrolled from both inpatient as well as outpatient settings, which is comparable to other studies from India ${ }^{(17,18)}$. Attia et $a l^{(19)}$ also studied children (aged 6-60 months) with complicated SAM and observed a low prevalence of rotavirus; about $8 \%$. However, they did not enrol any nonmalnourished controls. Ferdous et al. ${ }^{(16)}$ observed $30 \%$ prevalence of rotavirus diarrhoea in malnourished children and $33 \%$ in well-nourished children. However, they also included moderately malnourished children who may not have altered physiology and immune system as in children with SAM; this may explain the higher detection of rotavirus in their group of malnourished children. Das et $a l .{ }^{(20)}$, in their time-series modelling data over a period of 20 years (1993-2012), observed that reduction in proportion of underweight and wasting in Bangladesh was associated with a higher proportion of rotavirus infection as a cause of diarrhoea. In a longitudinal study among slumdwelling children from Bangladesh, Verkerke et al. (21) reported a significant association between better nutritional status and rotavirus infection in first 3 years of life. By a generalized mixed-effects model, they further derived that for each one-unit increment in height-for-age $Z$-score, 
weight-for-age $Z$-score and WHZ, the risk of rotavirus diarrhoea was higher by 36,32 and $18 \%$, respectively ${ }^{(21)}$. Findings from our study further consolidate the hypothesis that in malnourished children, diarrhoea is less likely to be due to rotavirus. The plausible mechanisms of rotavirus being less prevalent in malnourished children include immaturity of their gut microbiota ${ }^{(21,22)}$, rendering them more prone to bacterial infections; and presence of environmental enteropathy leading to shortening of the enterocytic villi ${ }^{(21,23)}$, which might be critical for rotavirus entry and replication. All these factors operate to a greater extent in children with more severe forms of malnutrition, which might explain a larger effect size in our study as compared with earlier studies, which evaluated differences between normal and moderately malnourished children.

Isolation of DEC was significantly higher $(P<0.001)$ in cases in comparison to controls. Similar high isolation rates were seen in previous other studies from our institution $^{(24,25)}$. Unregulated use of antibiotics disrupts normal flora and facilitates selective proliferation and colonization of DEC. We could not accurately enquire about antibiotic use due to recall bias, illiteracy of parents and lack of documentation of treatment by unqualified practitioners. Ferdous et $a l .{ }^{(16)}$ and Attia et $a l .{ }^{(19)}$ both found a lower prevalence of ETEC in malnourished children; 4.3 and $16 \%$, respectively. This may be due to the geographic variations between the studies. Ferdous et al. ${ }^{(16)}$ included children with moderate malnutrition and excluded children with dysentery from their study, which may explain the low isolation of ETEC. We detected Cryptosporidium in $9 \%$ of enrolled children. Similarly low isolation rate of Cryptosporidium was observed by Attia et al. ${ }^{(19)}$. These detection rates are comparatively lower than in earlier studies from similar settings ${ }^{(26-28)}$. Also, our results were not statistically significant between cases and controls because our study was not powered for Cryptosporidium detection. WHO estimates the incidence of persistent diarrhoea at about $10 \%$, which falls to $7 \%$ in community-based studies, and $20 \%$ of malnourished children develop persistent diarrhoea ${ }^{(29)}$. We observed low prevalence $(4 \%)$ of persistent diarrhoea in our study. Children in our study were managed as per treatment guidelines for SAM and diarrhoea, which could have led to lesser risk of persistent diarrhoea. We could follow-up 130 children; the mean number of episodes of diarrhoea in 3 months was comparable and not statistically significant between cases and controls. A relatively shorter duration of follow-up in our study and use of zinc supplementation and hygiene education could have nullified the difference between the two groups.

The main strength of our study was a comparative design, which enabled us to compare aetiological, anthropometric and clinical parameters between severely malnourished and non-malnourished children, and analyse for significant associations. We carried out the study in an urban hospital catering mainly to children from the lower socio-economic strata of society, where poor hygiene, improper sanitation and hand washing are still challenging, and management and prevention of diarrhoea are a priority. We carried out extensive stool microbiological work-up for aetiology of diarrhoea and tried to cover almost all of the common diarrhoeagenic pathogens in both our study groups, including antigen detection for rotavirus and Cryptosporidium and DNA extraction of E. coli and PCR for subtyping of $E$. coli.

The main limitation of our study was the hospital-based enrolment; and thus the results may not be representative of the patterns in the community setting. However, as it is recommended to hospitalize all children with SAM having diarrhoea, it may not be ethically possible to conduct such a study in the community setting, especially with follow-up for outcomes. Also, we did not enrol non-diarrhoeal controls in our study, which could have helped us to find out the asymptomatic carrier rate among our study groups and compare their disease-causing potential between the two groups. We calculated the sample size of our study according to rotavirus prevalence; this sample size was inadequate to enable comparison of prevalence of other organisms (with low isolation/detection rates) and risk of persistent diarrhoea, repeat episodes of diarrhoea and mortality. We could not perform molecular typing of rotavirus, which would have helped us to know the prevalent types in the two groups.

We conclude that rotavirus is a significantly lesser problem in children with SAM having acute diarrhoea compared with well-nourished children with diarrhoea, whereas DEC isolation is higher in children with SAM. This may suggest a change in paradigm from rotavirus to other diarrhoeagenic pathogens, especially in children with SAM. As SAM is a major risk factor of diarrhoeal mortality, our findings need to be confirmed in diverse community-based settings in low- and middle-income countries in order to prevent childhood mortality due to diarrhoea by formulating preventive and treatment strategies against the prevailing organisms.

\section{Acknowledgements}

Acknowledgements: The authors thank Dr Taru Singh (PhD), Department of Microbiology, University College of Medical Sciences, for help in carrying out molecular studies on the stool samples. Financial support: This work was partially funded by an intramural research grant from University College of Medical Sciences. University College of Medical Sciences had no role in design, analysis or writing of this article. Conflict of interest: None. Autborship: D.S. and P.G. conceptualized the study and finalized its design. A.J. collected the data and prepared the first draft of the manuscript. S.D. and R.S. handled the 
microbiological part of data collection and interpretation and provided critical inputs to manuscript preparation. D.S. and P.G. analysed the data and provided critical inputs to manuscript revision and its finalization. All authors approved the final version of manuscript and are accountable for all aspects of the work. Ethics of human subject participation: This study was conducted according to the guidelines laid down in the Declaration of Helsinki and all procedures involving human subjects/patients were approved by the Institutional Ethics Committee - Human Research (IEC-HR) of University College of Medical Sciences, Delhi. Written informed consent was obtained from the parents/guardians of all participants/patients.

\section{References}

1. World Bank (2017) Mortality rate, under-5 (per 1,000 live births). http://data.worldbank.org/indicator/SH.DYN.MORT (accessed April 2017)

2. Bassani DG, Kumar R, Awasthi S et al. (2010) Causes of neonatal and child mortality in India: a nationally representative mortality survey. Lancet 376, 1853-1860.

3. Kovacs SD, Mullholland K, Bosch J et al. (2015) Deconstructing the differences: a comparison of GBD 2010 and CHERG's approach to estimating the mortality burden of diarrhea, pneumonia, and their etiologies. BMC Infect Dis 15, 16.

4. Brown KH (2003) Diarrhea and malnutrition. J Nutr 133, issue 1, 328S-332S.

5. Savilahti E (1972) Immunoglobulin-containing cells in the intestinal mucosa and immunoglobulins in the intestinal juice in children. Clin Exp Immunol 11, 415-425.

6. Green F \& Heyworth B (1980) Immunoglobulin-containing cells in jejunal mucosa of children with protein-energy malnutrition and gastroenteritis. Arch Dis Child 55, 380-383.

7. Patwari AK (1999) Diarrhoea and malnutrition interaction. Indian J Pediatr 66, 1 Suppl., S124-S134.

8. Kotloff KL, Nataro JP, Blackwelder WC et al. (2013) Burden and aetiology of diarrhoeal disease in infants and young children in developing countries (the Global Enteric Multicenter Study, GEMS): a prospective, case-control study. Lancet 382, 209-222.

9. Kahn G, Fitzwater S, Tate J et al. (2012) Epidemiology and prospects for prevention of rotavirus disease in India. Indian Pediatr 49, 467-474.

10. World Health Organization \& UNICEF (2014) Progress on drinking water and sanitation: 2014 update. https://www. unicef.org/media/files/JMP_2014_Update.pdf (accessed April 2017).

11. Black RE, Brown KH \& Becker S (1984) Malnutrition is a determining factor in diarrheal duration, but not incidence, among young children in a longitudinal study in rural Bangladesh. Am J Clin Nutr 39, 87-94.

12. World Health Organization (2016) Diarrhoea. http://www. who.int/mediacentre/factsheets/fs330/en/ (accessed April 2017).
13. Dalwai S, Choudhury P, Bavdekar SB et al. (2013) Consensus Statement of the Indian Academy of Pediatrics on integrated management of severe acute malnutrition. Indian Pediatr 50, 399-404

14. Shah D \& Sachdev HPS (2012) Measuring undernutrition and overnutrition in children. In Public Health Nutrition in Developing Countries, pp. 108-150 [S Vir, editor]. New Delhi: Woodhead Publishing India Pvt. India.

15. World Health Organization (2014) Community-based management of severe acute malnutrition. https://www. who.int/maternal_child_adolescent/documents/a91065/en/ (accessed January 2017).

16. Ferdous F, Das SK, Ahmed S et al. (2013) Severity of diarrhea and malnutrition among under-five-year-old children in rural Bangladesh. Am J Trop Med Hyg 89, 223-228.

17. Mehendale S, Venkatasubramanian S, Kumar CG et al. (2016) Expanded Indian National Rotavirus Surveillance Network in the context of rotavirus vaccine introduction. Indian Pediatr 53, 575-581.

18. Kumar A, Basu S, Vashishtha V et al. (2016) Burden of rotavirus diarrhea in under-five Indian children. Indian Pediatr 53, 607-617.

19. Attia S, Versloot CJ, Voskuijl W et al. (2016) Mortality in children with complicated severe acute malnutrition is related to intestinal and systemic inflammation: an observational cohort study. Am J Clin Nutr 104, 1441-1449.

20. Das SK, Chisti MJ, Rahman Sarker MH et al. (2017) Long-term impact of changing childhood malnutrition on rotavirus diarrhoea: two decades of adjusted association with climate and socio-demographic factors from urban Bangladesh. PLoS One 12, e0179418.

21. Verkerke H, Sobuz S, Ma JZ et al. (2016) Malnutrition is associated with protection from rotavirus diarrhea: evidence from a longitudinal birth cohort study in Bangladesh. J Clin Microbiol 54, 2568-2574.

22. Subramanian S, Huq S, Yatsunenko T et al. (2014) Persistent gut microbiota immaturity in malnourished Bangladeshi children. Nature 510, 417-421.

23. Korpe PS \& Petri WA (2012) Environmental enteropathy: critical implications of a poorly understood condition. Trends Mol Med 18, 328-336.

24. Singh T, Das S, Ramachandran VG et al. (2017) Spectrum of diarrhoeagenic Escherichia coli in paediatric population suffering from diarrhoea and as commensals in healthy children. Indian J Med Microbiol 35, 204-210.

25. Singh T, Das S, Ramachandran VG et al. (2017) Distribution of integrons and phylogenetic groups among enteropathogenic Escherichia coli isolates from children $<5$ years of age in Delhi, India. Front Microbiol 8, 561-573.

26. Dabas A, Shah D, Bhatnagar S et al. (2017) Epidemiology of cryptosporidium in pediatric diarrheal illnesses. Indian Pediatr 54, 299-309.

27. Bera P, Das S, Saha R et al. (2014) Cryptosporidium in children with diarrhea: a hospital-based study. Indian Pediatr 51, 906-908.

28. Checkley W, White AC, Jaganath D et al. (2015) A review of the global burden, novel diagnostics, therapeutics, and vaccine targets for cryptosporidium. Lancet Infect Dis 15, 85-94.

29. Mathai J, Raju B \& Bavdekar A (2011) Chronic and persistent diarrhea in infants and young children: status statement. Indian Pediatr 48, 37-42. 